\title{
The physiotherapy management of patients undergoing abdominal surgery: A survey of current practice
}

Julie Reeve PhD, MSc, Grad Dip Phys

School of Clinical Sciences, Auckland University of Technology, Auckland, New Zealand

Lesley Anderson BHSC (Physiotherapy)

Senior Physiotherapist, Department of Physiotherapy, Waitemata District Health Board, Auckland, New Zealand

Yousef Raslan* BHSC (Physiotherapy)

School of Clinical Sciences, Auckland University of Technology, Auckland, New Zealand

Claudia Grieve* BHSC (Physiotherapy)

School of Clinical Sciences, Auckland University of Technology, Auckland, New Zealand

Jenna Ford* BHSC (Physiotherapy)

School of Clinical Sciences, Auckland University of Technology, Auckland, New Zealand

Livvy Wilson* BHSC (Physiotherapy)

School of Clinical Sciences, Auckland University of Technology, Auckland, New Zealand

*Undergraduate students at the time of the study

\section{ABSTRACT}

The aim of this study was to determine the physiotherapy management of patients undergoing upper and lower abdominal surgery performed via open or laparoscopic approaches in public hospital surgical units throughout New Zealand. The study also aimed to establish factors influencing physiotherapy practice and determine how New Zealand practice compares to current best evidence. A purpose-designed survey was distributed via post to the senior surgical ward physiotherapist of all public hospitals in New Zealand offering abdominal surgery $(n=23)$. A response rate of $83 \%(n=19)$ was obtained. No respondents reported routine physiotherapy input with patients prior to surgery. Only one surgical centre (5\%) provided prehabilitation, and this was to highrisk patients only. Postoperatively most respondents reported routine assessment and treatment of patients following open upper abdominal surgery, but not following open lower abdominal surgery or laparoscopic surgery. Early mobilisation and respiratory physiotherapy interventions were the most commonly implemented interventions postoperatively. This study identified that physiotherapy interventions for patients receiving abdominal surgery mainly focus on postoperative assessment and treatment of patients undergoing open upper abdominal surgery. Few units routinely assess these patients preoperatively or offer prehabilitation. This study will allow physiotherapists working with patients undergoing abdominal surgery in New Zealand to compare their own practice to that of others and consider their use of current best available evidence.

Reeve, J., Anderson, L., Raslan, Y., Grieve, C., Ford, J., \& Wilson, L. (2019). The physiotherapy management of patients undergoing abdominal surgery: A survey of current practice. New Zealand Journal of Physiotherapy, 47(2), 66-75. https://doi.org/10.15619/NZJP/47.2.02

Key Words: Abdominal Surgery, Survey, Physiotherapy Practice

\section{INTRODUCTION}

Abdominal surgery is the most common surgery undertaken in Australia and New Zealand (Australian Institute of Health and Wellness, 2017), and may involve a variety of major organs and structures such as the liver, pancreas, stomach, oesophagus or kidneys (Reeve \& Boden, 2016). Upper abdominal surgery (UAS) is defined as an incision above or extending above the umbilicus. Lower abdominal surgery (LAS) is defined as an incision below the umbilicus. Abdominal surgery may be open (involving an incision greater than $5 \mathrm{~cm}$ ), laparoscopic (usually involving several small incisions of approximately $0.5-2 \mathrm{~cm}$ ) or a combination of both. Initially, laparoscopic surgeries were widely undertaken for some lower risk abdominal surgeries. Typically, these were associated with less pain, shorter recovery times, a shorter length of hospital stay and reduced complication rates compared with open abdominal surgeries (Spanjersberg, van Sambeeck, Bremers, Rosman, \& van Laarhoven, 2015; Veldcamp et al., 2005). However, more recent surgical advances have improved the ability to undertake more complex surgeries via laparoscope or hand-assisted laparoscopic surgeries (HALS), also known as minimally invasive surgery. In HALS an additional incision allows a hand to be passed into the abdomen for surgical manipulation and tissue removal. Whilst these surgeries usually demonstrate accelerated recovery times, a shorter length of stay in hospital and reductions in postoperative complication rates (Benlice, Costedio, Stocchi, Abbas, \& Gorgun, 2016; 
Spanjersberg et al., 2015), they often involve longer anaesthetic times compared with their equivalent open procedure (Owen et al., 2013).

Physiotherapy management of patients following major abdominal surgery aims to reduce postoperative complications and encourage early postoperative activity to avoid the sequelae of surgery and immobility (Hanekom et al., 2012; Makhabah, Martino, \& Ambrosino, 2013). Recently there have been several high-quality studies which have better defined the efficacy of physiotherapy interventions for patients undergoing abdominal surgery (see Reeve \& Boden, 2016 for a review of this evidence). However, it is not currently known what comprises physiotherapy interventions for such patients in New Zealand and whether this follows current best evidence recommendations. Given the changes in surgical techniques, it is timely to determine if and how these changes have impacted on physiotherapy practice in this population. Therefore, the aim of this study was to establish the current preoperative and postoperative physiotherapy management of patients undergoing all abdominal surgery and surgical approaches across public hospital surgical units in New Zealand. The study also aimed to establish what factors influence physiotherapy service provision for patients undergoing abdominal surgery in New Zealand and to contextualise this with current best available evidence.

\section{METHODS}

This study targeted all public hospitals in New Zealand offering abdominal surgery. Hospitals were identified from the New Zealand Ministry of Health website (www.health.govt.nz), and 37 public hospitals offering surgical treatment were identified. Telephone contact to each of these hospitals was required to identify those that specifically offered upper, lower, open and/or laparoscopic abdominal surgery. In total, 26 public hospitals were found to offer abdominal surgery and thus were approached to take part in the study.

At the time of this study no survey had previously been designed or evaluated that investigated the physiotherapy interventions for patients undergoing abdominal surgery. Therefore, a survey was purpose-designed for the task. The survey comprised 48 questions and was divided into four sections: demographic information, service provision, preoperative physiotherapy management and postoperative physiotherapy management. The majority of questions in the survey were closed questions, with a small number of open questions utilised to give respondents the opportunity to comment or give specifics where appropriate. The survey was developed by two senior respiratory physiotherapists and two student physiotherapists as part of a BHSc (Physiotherapy) student research project. The survey was piloted by two practising physiotherapists with experience managing patients undergoing abdominal surgery. As a result, minor amendments were made to clarify wording, reduce ambiguity and improve the flow of the survey. The study and survey received ethical approval from the Auckland University of Technology Ethics Committee (ref: 17/10) and was administered in April 2017. Completion and return of the survey were taken to indicate informed consent.
A cover letter explaining the purpose of the survey, a survey and a return envelope were posted to each of the identified hospitals, addressed to the senior physiotherapist of the acute/ surgical ward. The most senior physiotherapist involved in the management of adults undergoing abdominal surgery was asked to complete the survey in accordance with general practice at their centre. If no senior physiotherapist was available, then any physiotherapist involved in the care of patients undergoing abdominal surgery could complete the survey. Respondents were requested to return the survey within three weeks. Surveys were coded to enable the research team to follow up any non-responders after this time, and repeat surveys were sent to non-responders. Following this, all surveys were de-identified and non-responders were not approached further. In instances where the hospital did not offer physiotherapy management but did offer abdominal surgery, respondents were asked to return the survey stating no physiotherapy was offered to their patients undergoing abdominal surgery.

\section{Data analysis}

All closed question response data were of the nominal/ordinal form and analysed using Microsoft Excel 2016. Descriptive statistics were used to summarise findings, and categorical variables were summarised as frequencies and percentages. Open questions were analysed by content analysis and development of themes. Content analysis was undertaken by summarising responses into a series of single themes for each open question.

\section{RESULTS}

\section{Response rate}

A total of 26 surveys were distributed to public hospitals in New Zealand identified as potentially offering abdominal surgery according to our initial search strategy. Two surveys were returned by physiotherapists stating that their units did not offer abdominal surgery and one respondent indicated that their unit provided no physiotherapy services to patients undergoing abdominal surgery; thus, 23 public hospitals were eligible to take part in the survey. Of these, $83 \%(n=19)$ returned completed surveys. The results presented below are calculated from those who responded to the survey $(n=19)$.

\section{Demographics}

Of the 19 respondents that completed the survey, the majority of physiotherapists ( $n=12 ; 63 \%$ ) reported completing their undergraduate physiotherapy qualification over 10 years ago and $13(68 \%)$ respondents reported having between one and five years' clinical experience as a senior physiotherapist on surgical wards.

All respondents reported open and laparoscopic UAS and LAS surgery was performed in their surgical centre. Hand-assisted laparoscopic UAS and LAS was performed in eight (42\%) surgical centres. All respondents $(n=19,100 \%)$ reported that upper gastrointestinal (UGI) and colorectal ( $n=19 ; 100 \%)$ surgery was performed. The types of abdominal surgery undertaken in hospitals in New Zealand are shown in Figure 1.

Enhanced recovery after surgery (ERAS) protocols were reported to be utilised in 15 (79\%) centres following abdominal surgery. 


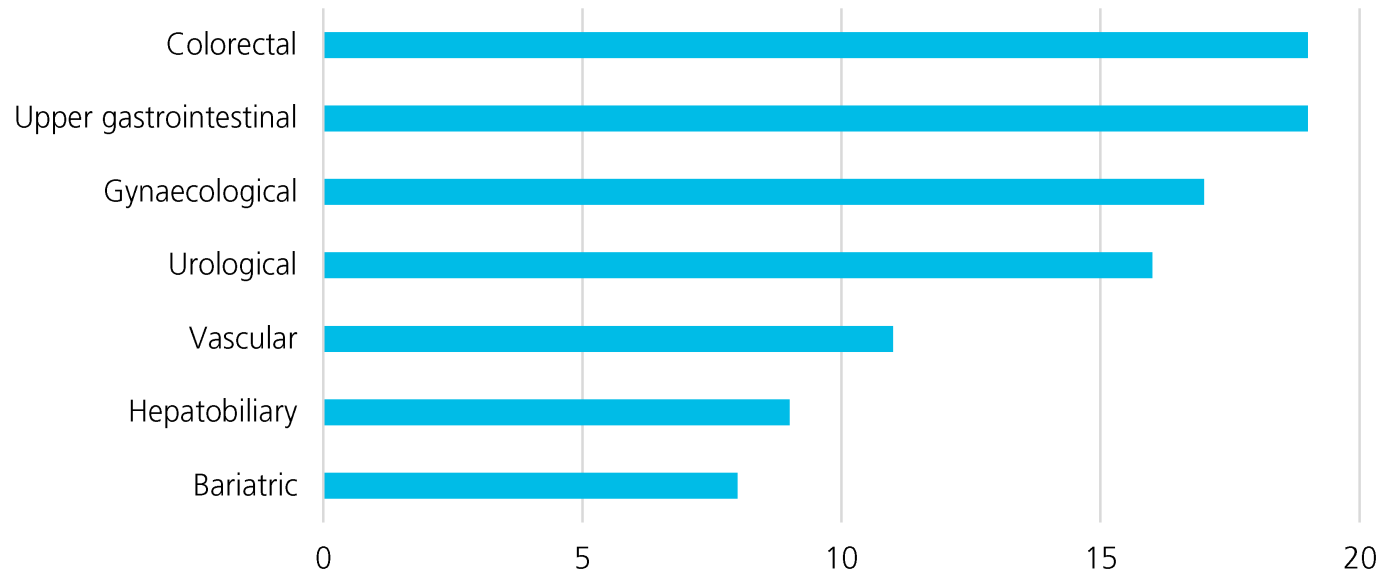

number of respondents $(n=19)$

Figure 1: Types of abdominal surgeries offered in hospitals in New Zealand

Physiotherapy service provision to patients undergoing abdominal surgery

Two (11\%) respondents reported having one or fewer full-time equivalent (FTE) physiotherapists staffing the abdominal surgical wards daily; 15 (79\%) reported having between one and four FTE physiotherapists; and two (11\%) reported between five and eight FTE physiotherapists. In all cases, this excluded weekend service provision.

Table 1 shows the number of respondents who reported providing a weekend, evening and/or overnight (out of normal work hours) physiotherapy service for patients following abdominal surgery. Only one respondent (5\%) reported that they did not provide any weekend, evening or overnight service for patients following abdominal surgery.

Preoperative physiotherapy management for patients undergoing abdominal surgery

No respondents reported assessing all patients undergoing abdominal surgery preoperatively, with most reporting that they did not assess any patients preoperatively $(n=11$;
$58 \%)$. This was due to a variety of reasons. However, most of the respondents that reported not assessing any patients preoperatively stated this was due to insufficient time or resources $(n=7 ; 37 \%)$ or that preoperative education in their centres was delivered by nurses and/or information leaflets ( $\mathrm{n}=$ 5; $26 \%$ ). Two respondents (11\%) reported they were about to commence a preoperative physiotherapy service.

Eight respondents (42\%) reported seeing "some" patients who had been referred to physiotherapy by the surgeon or anaesthetist preoperatively, and that generally these patients were selected because they were considered at high risk of developing postoperative complications. High-risk patients were considered by respondents as having complex comorbidities, open UGI surgery, chronic respiratory disease, or other concerns identified by the medical team. Respondents reported assessing these referred patients at pre-admission clinic visits. Risk prediction tools were reported to be utilised by two respondents $(11 \%)$ to identify those patients at high risk of developing a postoperative pulmonary complication although neither respondent named the tool utilised for risk prediction.

Table 1: Types of services offered for patients following abdominal surgery $(n=19)$

\begin{tabular}{lccc}
\hline Service offered & $\begin{array}{c}\text { Overnight } \\
\mathrm{n}(\%)\end{array}$ & $\begin{array}{c}\text { Evening } \\
\mathrm{n}(\%)\end{array}$ & $\begin{array}{c}\text { Weekend } \\
\mathrm{n}(\%)\end{array}$ \\
\hline Yes, as routine service & $0(0)$ & $0(0)$ & $7(37)$ \\
Yes, but only prn & $1(5)$ & $1(5)$ & $10(53)$ \\
On-call service only & $10(53)$ & $6(32)$ & $1(5)$ \\
No service & $6(32)$ & $2(11)$ & $1(5)$ \\
Missing data & $2(11)$ & $0(0)$ \\
\hline
\end{tabular}

Notes: n, number; prn, as required 
Of those respondents that assessed "some" patients preoperatively ( $n=8,42 \%$ ), preoperative education and advice was identified as the main intervention administered. All eight respondents stated education was delivered via face-to-face contact, and three respondents reported additionally providing an information booklet. Those providing preoperative education and advice included education in undertaking effective breathing exercises postoperatively $(n=7)$ and chest clearance techniques $(n=5)$. Three $(16 \%)$ respondents reported also providing "brief" preoperative exercise advice.

Most respondents offered no prehabilitation services ( $n=18$, $95 \%$ ). However, one respondent (5\%) reported providing exercise prehabilitation occasionally to selected high-risk patients. This programme commenced within two weeks prior to surgery and comprised home-based aerobic exercise training once to twice weekly. A further respondent (5\%) stated they had the ability to refer patients for prehabilitation to an off-site clinic, as no prehabilitation was administered in their centre. No respondents reported offering inspiratory muscle training programmes to patients undergoing abdominal surgery.

Postoperative physiotherapy management for patients undergoing abdominal surgery

Figure 2 identifies the type of abdominal surgical procedures where postoperative physiotherapy assessment and treatment was reported to be routinely administered.

Physiotherapy management following open UAS Seventeen respondents (89\%) reported assessing all patients following open UAS, while 10 respondents (53\%) reported implementing prophylactic physiotherapy treatment interventions with all patients postoperatively. Physiotherapy assessment and treatment commenced on postoperative day one (POD1) in all cases. No respondent reported assessing or treating patients on the day of surgery. Two respondents (11\%) reported that they did not routinely assess or treat any patients following open UAS but relied on referral from other medical team members.

Of those patients in surgical centres where postoperative physiotherapy assessment was routinely undertaken $(n=17)$, patients were usually assessed once daily ( $n=12 / 17 ; 71 \%)$. Respondents were asked to indicate prophylactic interventions that they considered they would normally use or not normally use with patients having undergone uncomplicated open UAS (Table 2).

Both nurses and physiotherapists reported routinely assisting patients to sit out of bed and subsequent ambulation interventions. However, respondents reported physiotherapists were the discipline to usually initiate ambulation interventions, and these commenced on POD1 where possible. Exercise interventions, the staff involved and the usual day of commencement are shown in Table 3.

\section{Physiotherapy following open LAS, HALS and laparoscopic surgery}

Seven respondents (37\%) stated that their postoperative care of patients undergoing open LAS did not differ to their management of those undergoing open UAS. Seven respondents $(37 \%)$ reported physiotherapists were not normally involved in the postoperative care of patients undergoing open LAS. The remaining respondents $(n=5,26 \%)$ reported that open LAS patients were only seen by a physiotherapist if they were considered high-risk, otherwise nursing staff managed all postoperative care.

Following laparoscopic surgery, three respondents (16\%) reported that physiotherapists prophylactically assessed and treated patients undergoing upper abdominal laparoscopic or HALS. All other respondents $(n=16,84 \%)$ reported no involvement in either UAS or LAS via laparoscope or HALS.

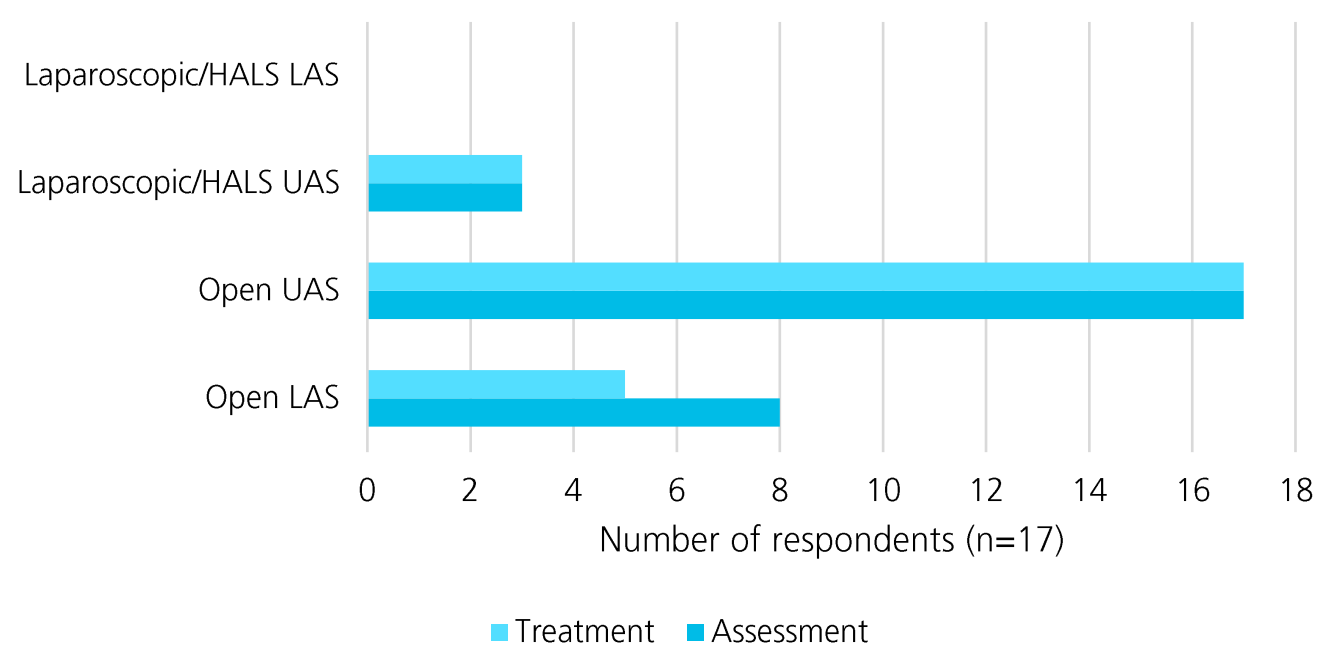

Notes: HALS, hand assisted laparoscopic surgery; UAS, upper abdominal surgery; LAS, lower abdominal surgery.

Figure 2: Abdominal surgical approaches where physiotherapists provide routine postoperative assessment and treatment 
Table 2: Prophylactic physiotherapy interventions routinely used by physiotherapists in the management of patients undergoing uncomplicated open upper abdominal surgery $(n=17)$

\begin{tabular}{lcc}
\hline Treatment intervention & $\begin{array}{c}\text { Normally utilised } \\
\text { postoperatively } \\
\mathrm{n}(\%)\end{array}$ & $\begin{array}{c}\text { Not normally utilised } \\
\text { postoperatively } \\
\text { (\%) }\end{array}$ \\
\hline Supervised mobilisation & $17(100)$ & $0(0)$ \\
Deep breathing exercises* & $16(94)$ & $0(0)$ \\
Cough/supported cough & $16(94)$ & $1(6)$ \\
ACBT & $13(76)$ & $4(24)$ \\
Forced expiratory technique & $12(71)$ & $5(29)$ \\
Incentive spirometry & $4(24)$ & $13(76)$ \\
PEP devices & $2(12)$ & $15(88)$ \\
Manual chest techniques e.g. clapping, shaking, vibrations & $0(0)$ & $17(100)$ \\
Non-invasive ventilation* & $0(0)$ & $16(94)$
\end{tabular}

Notes: ACBT, active cycle of breathing techniques; $n$, number; PEP, positive expiratory pressure; ${ }^{*} n=16$

Table 3: Exercise interventions undertaken following open upper abdominal surgery; staff undertaking them; and the postoperative day commenced $(n=17)$

\begin{tabular}{lcccc}
\hline Intervention & $\begin{array}{c}\text { Physiotherapist-led } \\
\mathrm{n}(\%)\end{array}$ & $\begin{array}{c}\text { Nurse-led } \\
\mathrm{n}(\%)\end{array}$ & $\begin{array}{c}\text { Not routinely undertaken } \\
\mathrm{n}(\%)\end{array}$ & $\begin{array}{c}\text { Postoperative day commenced } \\
\text { Mode (range) }\end{array}$ \\
\hline Lower limb exercises & $5(26)$ & $1(5)$ & $10(53)$ & $1(0-2)$ \\
Sitting out of bed & $13(77)$ & $15(88)$ & $0(0)$ & $0(0)$ \\
Ambulation & $17(100)$ & $11(65)$ & $4(24)$ & $1(1)$ \\
Stair climbing & $13(77)$ & $0(0)$ & $3(18)$ & Pre-discharge (3-7) \\
$\begin{array}{l}\text { Discharge exercises and } \\
\text { advice booklet }\end{array}$ & $11(65)$ & $4(24)$ & & Day of discharge (1-4) \\
\hline
\end{tabular}

Note: n, number

\author{
Use of allied health assistants following abdominal \\ surgery \\ Sixteen (84\%) respondents reported using allied healthcare \\ assistants to assist with undertaking postoperative mobilisation \\ with patients; five respondents (26\%) reported they also \\ utilised healthcare assistants to assist in undertaking respiratory \\ interventions. All stated healthcare assistants became involved \\ when the patient was determined to be medically stable but \\ had not reached preadmission respiratory status and/or an \\ acceptable mobility level. \\ Discharge criteria \\ Table 4 outlines criteria that respondents reported must be \\ achieved prior to being discharged from physiotherapy following \\ abdominal surgery.
}

Factors influencing physiotherapy management for patients undergoing abdominal surgery Respondents were given statements to reflect upon regarding their translation of research findings into clinical practice (Table $5)$.

Respondents were also asked to rank which factors influence their physiotherapy management via a Likert Scale (1 "no influence at all" to 5 "very influential"). The results are shown in Table 6.

\section{DISCUSSION}

The excellent response rate to this survey (83\%) suggests the results are highly likely to be representative of the population studied (Fincham, 2008). The results of this study document 
Table 4: Physiotherapy management prior to discharge from physiotherapy $(n=19)$

\begin{tabular}{lc}
\hline Discharge criteria & $\mathrm{n}(\%)$ \\
\hline Independent with secretion clearance OR non-productive of sputum & $19(100)$ \\
Stable respiratory status (SpO2, RR, CXR) & $18(95)$ \\
Mobilising at preadmission level of mobility & $9(47)$ \\
Independent with activities of daily living e.g. showering, toileting & $4(21)$ \\
\hline
\end{tabular}

Notes: CXR, chest $x$-ray; $n$, number; RR, respiratory rate; SpO2, peripheral oxygen saturation

Table 5: Self-determined familiarity with the research evidence base $(n=19)$

\begin{tabular}{ll}
\hline Statement & $\mathrm{n}(\%)$ \\
\hline I use the literature regularly to influence my practice and can name the studies I use & $2(11)$ \\
I feel very familiar with the literature but am unable to specifically name the studies which have & $9(47)$ \\
influenced this & $4(21)$ \\
I am familiar with the literature but other factors influence my practice rather than the literature & $4(21)$ \\
\hline
\end{tabular}

Note: $n$, number

Table 6: Participant ranking of factors influencing physiotherapy practice $(n=19)$

\begin{tabular}{|c|c|c|c|c|c|}
\hline $\begin{array}{l}\text { Influencing factor } \\
\mathrm{n}(\%)\end{array}$ & $\begin{array}{c}1 \\
\text { (no influence) }\end{array}$ & 2 & $\begin{array}{c}3 \\
\text { (neutral) }\end{array}$ & 4 & $\begin{array}{c}5 \\
\text { (very influential) }\end{array}$ \\
\hline Literature recommendation & $0(0)$ & $0(0)$ & $3(16)$ & $12(63)$ & $4(21)$ \\
\hline Personal experience & $0(0)$ & $1(5)$ & $1(5)$ & $9(47)$ & $8(42)$ \\
\hline Unit policy/protocol & $1(5)$ & $2(11)$ & $6(32)$ & $5(26)$ & $5(26)$ \\
\hline Surgical colleagues' preferences* & $2(11)$ & $6(32)$ & $1(5)$ & $6(32)$ & $3(16)$ \\
\hline Financial and/or resource constraints & $2(11)$ & $1(5)$ & $4(21)$ & $7(37)$ & $5(26)$ \\
\hline
\end{tabular}

Notes: $n$, number; ${ }^{\star} \mathrm{n}=18$

physiotherapy practice for patients undergoing abdominal surgery in New Zealand. Results will allow New Zealand physiotherapists to benchmark their service provision and interventions with other centres in New Zealand and overseas (Patman, Bartley, Ferraz, \& Bunting, 2017), and against best practice recommendations (Hanekom et al., 2012; Reeve \& Boden, 2016). The results will also provide a platform from which to further investigate the physiotherapy management of adults undergoing abdominal surgery in New Zealand.

This survey demonstrated that physiotherapists from all responding surgical centres were routinely involved with patients undergoing UAS, but few were routinely involved with those undergoing open LAS. This practice is likely to be based on evidence that suggests PPCs are 15 times more likely and that pneumonia is almost three times more likely following UAS compared with LAS (Arozullah, Khuri, Henderson \& Daley, 2001; Smith et al., 2010). It also reflects recently published recommendations that routine physiotherapy following LAS is unnecessary (Reeve \& Boden, 2016) providing that, where necessary, high-risk patients are assessed to determine the risk/ presence of PPC and treated as appropriate.

Our study also demonstrated that prophylactic physiotherapy interventions were not usually undertaken with patients undergoing laparoscopic surgery and HALS. Typically, laparoscopic surgery and HALS have accelerated recovery times, reduced complication rates and a shorter length of hospital stay, with the incidence of PPCs after traditional laparoscopic surgery being negligible (Antoniou, Antoniou, Koch, Pointner, \& Granderath, 2014; Smith et al., 2010; Spanjersberg et al., 2015). Additionally, physiotherapy following laparoscopic surgery has not been shown to improve outcomes (Fagevik Olsén, Josefson, \& Lönroth, 1999). However, given the increasing complexity, duration and types of major surgeries (such as hepatic, oesophageal, pancreatic and colorectal 
surgeries) being undertaken by more advanced laparoscopic and HALS procedures, there is a need to re-evaluate the prevalence of postoperative complications amenable to physiotherapy; and to determine whether physiotherapy interventions following such surgeries improves outcomes. Until further contemporary data are available, it is recommended that physiotherapists undertake risk assessment of patients undergoing advanced laparoscopic surgery and initiate treatment where necessary.

\section{Preoperative education}

The purpose of preoperative physiotherapy education is to improve patient knowledge regarding rehabilitation required in the postoperative recovery period; to teach postoperative exercises to reduce postoperative complication risk; and to enhance patient's recovery to their desired level of function postoperatively. In previous decades physiotherapy preoperative education was, anecdotally, widely provided for patients undergoing major surgery, but more recently, physiotherapy practices have primarily focused on postoperative education (Patman, 2018). In our survey, most respondents reported not providing any preoperative physiotherapy education to patients undergoing abdominal surgery, primarily due to resourcing issues. Surveys in Australia have shown similar findings in relation to preoperative practices (Browning, Denehy, \& Scholes, 2007; Patman et al., 2017; Scholes, Denehy, Sztendur, \& Browning, 2006) despite an accumulating body of evidence demonstrating preoperative physiotherapy education reduces the incidence of PPC in patients undergoing open UAS (Boden et al., 2018; Fagevik Olsén, Hahn, Nordgren, Lönroth, \& Lundholm, 1997; Samnani, Umer, Mehdi, \& Farid, 2014). Moreover, the largest and most recent of these was a multicentre, international randomised controlled trial undertaken in two Australian centres and one New Zealand centre. This trial delivered a single 30-minute preoperative face-to-face education session and provided a booklet within a hospital multidisciplinary preadmission clinic within six weeks of open UAS. The intervention halved the incidence of PPCs in the treatment group when compared with a control group who received the booklet alone without face-to-face education (Boden et al., 2018). Together with the previous studies, Boden et al.'s (2018) study presents robust evidence that preoperative education delivered by a physiotherapist within six weeks of major open UAS improves outcomes and should be a recognised evidence-based intervention in patients undergoing this type of surgery. Given the likely low cost of this intervention compared with the costs associated with PPC development (Shander et al., 2011), we would recommend New Zealand surgical centres consider implementing such interventions for patients undergoing major open abdominal surgery.

\section{Prehabilitation}

With the increasing focus on prevention rather than cure, there has been developing interest over the last decade in investigating the efficacy of utilising preoperative exercise interventions (prehabilitation) to improve surgical outcomes. Prehabilitation aims to improve functional capacity and cardiopulmonary fitness to enable patients to withstand the stresses of surgery, and reduce postoperative morbidity and mortality (West, Wischmeyer, \& Grocott, 2017). Typically, prehabilitation provides preoperative exercise-based interventions (unimodal), but more recently models have changed to include medical, nutritional, behavioural and psychosocial optimisation (bi/trimodal models). Whilst the rationale for prehabilitation is widely accepted, in practice the implementation of these programmes in patients with often short surgical waitlist times and complex neoadjuvant therapies can be challenging. In New Zealand, only one surgical centre in this survey reported implementing an exercise-based prehabilitation programme, and this was implemented for only "some" high-risk patients. There is clear evidence of the impact of poor preoperative cardiorespiratory fitness on postoperative outcome (West, Lythgoe, et al., 2014; West, Parry, et al., 2014) and of the impact of prehabilitation on preoperative cardiorespiratory fitness (Loughney, West, Kemp, Grocott, \& Jack, 2015; Snowdon, Haines, \& Skinner, 2014; West et al., 2015). However, the evidence for this translating into improved postoperative outcomes is yet to be conclusively demonstrated (Hijazi, Gondal, \& Aziz, 2017; O'Doherty, West, Jack, \& Grocott, 2013; West et al., 2017). Similarly, whilst the research investigating the efficacy of prehabilitation in improving outcomes for patients undergoing abdominal surgery is extensive (to date there are approximately 19 systematic reviews), this same research is limited by the heterogeneity seen in the populations being investigated; the type, frequency, duration and timing of interventions; and in the uni-, bi-, or tri-modal approaches to prehabilitation. Additionally, there are numerous methodological limitations to these studies, such as small sample sizes and low-quality study designs. Furthermore, the lack of standardisation of the outcome measures makes interpretation of the results challenging. Indeed, only a handful of studies have attempted meta-analysis of data due to the heterogeneity of the studies included in the systematic reviews (Kendall, Oliveira, Peleteiro, Pinho, \& Bastos, 2018; Loughney et al., 2015; Moran et al., 2016). Thus, despite a rapidly growing body of literature, to date no firm conclusions can be drawn regarding the efficacy of prehabilitation in improving surgical outcomes for patients undergoing abdominal surgery. In general, authors suggest that exercise prehabilitation should be offered to all patients undergoing elective major or complex abdominal surgery, and where resources are scarce, this should be limited to those at high risk of perioperative complications (Hijazi et al., 2017; Reeve \& Boden, 2016; Tew, Ayyash, Durrand, \& Danjoux, 2018).

\section{Postoperative physiotherapy}

The role of the physiotherapist in the postoperative period is to prevent postoperative complications (particularly PPCs) and facilitate return to the desired level of function (Reeve \& Boden, 2016). The current study found that most physiotherapists in New Zealand public surgical units routinely assessed and prophylactically treated patients following open UAS. Routine interventions consisted of deep breathing and coughing exercises $(D B \& C)$, and supervised ambulation interventions, normally commencing on POD1. The use of prophylactic respiratory interventions in the early postoperative stage is consistent with physiotherapy service provision in other countries and expert consensus (Hanekom et al., 2012; Patman et al., 2017), despite the necessity for DB\&C interventions remaining contentious across different major surgical groups (Pasquina, Tramèr, \& Walder, 2003; Reeve et al., 2010), 
including those undergoing UAS (Mackay, Ellis, \& Johnston, 2005; Pasquina, Tramèr, Granier, \& Walzer, 2006; Silva, Li, \& Rickard, 2013). For example, in patients undergoing UAS, two studies (Mackay et al., 2005; Silva et al., 2013) have demonstrated that the addition of deep breathing exercises to physiotherapy-directed early (POD1) postoperative ambulation does not further reduce PPCs compared with early postoperative ambulation alone. This suggests that early postoperative ambulation on POD1 may be all that is necessary in low-risk patients, and that the routine use of prophylactic DB\&C may not be justified. Despite the uncertain evidence regarding the use of DB\&C in this population and given the potential cost-versusharm benefits of implementing these interventions, guidelines currently continue to suggest that DB\&C exercises continue to be employed (Hanekom et al., 2012).

In the current study, the widespread initiation by physiotherapists of supervised ambulation in the early postoperative period in patients following abdominal surgery mirrors recommendations from the literature to utilise such interventions to prevent the deleterious impact of prolonged immobility. Most respondents reported initiating these interventions once daily, commencing on POD1. Our results regarding the widespread use of ambulation interventions by physiotherapists may reflect that enhanced recovery after surgery (ERAS) protocols are reportedly widely utilised in New Zealand surgical centres, and that patients managed within ERAS protocols mobilise more frequently and achieve independent mobilisation earlier than those cared for without ERAS (Basse, Hjort-Jakobsen, Billesbølle, Werner, \& Kehlet, 2000). This occurs despite the early mobilisation component being the least adhered to of all the ERAS components (Boulind et al., 2012; Gustafsson et al., 2011); that ERAS pathways and mobilisation goals differ between centres (Basse et al., 2000); and that patients may perform little ambulation outside of physiotherapy-directed ambulation in the first few postoperative days (Browning et al., 2007). One study investigating the impact of delayed ambulation found that for each postoperative day that patients did not mobilise greater than $10 \mathrm{~m}$ from the bed, they were three (95\% CI 1.2 to 8) times more likely to develop a PPC (Haines et al., 2013). A recent study demonstrated that in patients following colorectal surgery, missing supervised ambulation interventions in the early postoperative period correlated with an increased length of stay in hospital, and that participating in three supervised ambulation sessions each day commencing on POD1 predicted a reduced length of stay (Stethen et al., 2018).

Despite the protocolisation of ambulation found within ERAS paradigms, few respondents in our study reported ambulating patients to a specific target level of exertion, intensity, time or distance. Whilst some studies have shown better outcomes to be associated with standardised, structured approaches to the implementation of ambulation protocols early in the postoperative period (Pashikanti \& Von Ah, 2012), the evidence for this remains limited and no uniform definition of what constitutes early mobilisation/ambulation currently exists for patients following abdominal surgery (Feldheiser et al., 2016). Recently, one such standardised protocol was nested within a high-quality randomised controlled trial in 441 patients undergoing UAS (which included patients in New Zealand) (Boden et al., 2018). This protocol used a postoperative physiotherapy-directed standardised ambulation programme commencing on POD1 and found the median (IQR) time to achieve ambulation for over 10 minutes following open UAS was three (IQR 1-5) days and, importantly, that there were no adverse events associated with this level of ambulation. To date, the optimal frequency, duration and intensity levels of ambulation to reduce postoperative complications have yet to be determined, and until further robust evidence is available, it is suggested that a standardised protocol similar to Boden et al.'s (2015) protocol for early ambulation is followed, as this has been demonstrated to be safe and achievable in New Zealand surgical populations

Most of the respondents to our study reported that alongside personal experience, utilising evidence-based practice was a strong influencing factor on their practice. Overall, findings from this survey have indicated that physiotherapists in surgical centres in New Zealand adhere to evidence-based guidelines for managing patients undergoing abdominal surgery postoperatively. However, while there is an increasing body of evidence suggesting interventions such as preoperative education (Boden et al., 2018; Fagevik Olsén et al., 1997; Samnani et al., 2014) and prehabilitation exercise interventions (Katsura, Kuriyama, Takeshima, Fukuhara, \& Furukawa, 2015; Mans, Reeve, \& Elkins, 2015; Moran et al., 2016; Tew et al., 2018) may be effective in reducing postoperative complications and enhancing postoperative recovery, to date, this does not appear to have impacted upon clinical practice. Whilst the contemporary nature of some of this research may be one reason for the delay in translation of evidence to practice, other potential reasons should be identified and addressed where necessary.

\section{Limitations}

This study sought to obtain the relevant information from one physiotherapist per abdominal surgical centre in New Zealand, and we acknowledge that opening this survey to all physiotherapists in each centre might have resulted in differing responses. We tried to alleviate this potential variability by targeting the senior physiotherapist in each centre, anticipating that this person was most likely to be determining practice in the surgical centre. Additionally, open questions offered the possibility for respondents to comment on the diversity of practice in each centre. We acknowledge that this survey was administered only to public hospitals in New Zealand and that responses from physiotherapists working in private hospital abdominal surgical centres may differ. Of the 36 private hospitals identified through New Zealand databases, a significant proportion of these offered day surgery or minimally invasive surgery only, and this, together with different funding models, drove our decision to omit private hospitals from this survey.

\section{Clinical implications}

This study will allow physiotherapists working in abdominal surgical units around New Zealand to compare their preoperative and postoperative management of patients undergoing abdominal surgery to other similar centres, both in New Zealand and overseas (Patman et al., 2017). It is hoped that this study will assist practitioners to evaluate their practice 
in accordance with current evidence, implement changes where appropriate and audit the impact of implementing such changes.

\section{CONCLUSION}

This study has determined the current physiotherapy management of patients undergoing abdominal surgery in public hospitals throughout New Zealand. It has identified common physiotherapy interventions that are utilised with these patients in preoperative and postoperative settings, and the similarities and differences in practice as well as factors that influence current practice. It has compared current New Zealand practices to that of the most recent literature, thus allowing physiotherapists to evaluate their own practice regarding best available evidence.

\section{KEY POINTS}

1. Most physiotherapists in surgical centres in New Zealand adhere to evidence-based guidelines for the postoperative inpatient management of patients undergoing open upper abdominal surgery.

2. Physiotherapists working in New Zealand surgical centres are rarely routinely involved in the management of patients undergoing open lower abdominal surgery or laparoscopic surgery.

3. Prior to their surgery, people undergoing abdominal surgery in New Zealand rarely receive education and advice or prehabilitation interventions from physiotherapists.

\section{DISCLOSURES}

No funding was obtained for this study. There are no conflicts of interest which may be perceived to interfere with or bias this study.

\section{PERMISSIONS}

Ethical approval was gained from the Auckland University of Technology Ethics Committee (ref: 17/10).

\section{ADDRESS FOR CORRESPONDENCE}

Dr Julie Reeve, Department of Physiotherapy, School of Clinical Sciences, Auckland University of Technology, Private Bag 92006, Auckland, New Zealand. Telephone: +64 99219999 ext. 7085. Email: julie.reeve@aut.ac.nz.

\section{REFERENCES}

Antoniou, S. A., Antoniou, G. A., Koch, O. O., Pointner, R., \& Granderath, F. A. (2014). Meta-analysis of laparoscopic vs open cholecystectomy in elderly patients. World Journal of Gastroenterology, 20(46), 17626-17634. https://doi.org/10.3748/wjg.v20.i46.17626

Arozullah, A. M., Khuri, S. F., Henderson, W. G., Daley, J.; Participants in the National Veternas Affairs Surgical Quality Improvement Program. (2001). Development and validation of a multifactorial risk index for predicting postoperative pneumonia after major noncardiac surgery. Annals of Internal Medicine, 135(10), 847-857. https://doi.org/10.7326/0003-4819135-10-200111200-00005

Australian Institute of Health and Wellness. (2017). Procedures and healthcare interventions (ACHI 9th edition) 2015-16 to 2016-17. Retrieved from https://www.aihw.gov.au/reports/hospitals/procedures-data-cubes/ contents/data-cubes
Basse, L., Hjort-Jakobsen, D., Billesbølle, P., Werner, M., \& Kehlet, H. (2000). A clinical pathway to accelerate recovery after colonic resection. Annals of Surgery, 232(1), 51-57. https://doi.org/10.1097/00000658-20000700000008

Benlice, C., Costedio, M., Stocchi, L., Abbas, M. A., \& Gorgun, E. (2016). Hand-assisted laparoscopic vs open colectomy: An assessment from the American College of Surgeons National Surgical Quality Improvement Program procedure-targeted cohort. American Journal of Surgery, 212(5), 808-813. https://doi.org/10.1016/j.amjsurg.2016.02.014

Boden, I., Browning, L., Skinner, E. H., Reeve, J., El-Ansary, D., Robertson, I. K., \& Denehy, L. (2015). The LIPPSMAck POP (Lung Infection Prevention Post Surgery - Major Abdominal - with Pre-Operative Physiotherapy) trial: Study protocol for a multi-centre randomised controlled trial. Trials, 16, 573. https://doi.org/10.1186/s13063-015-1090-6

Boden, I., Skinner, E., Browning, L., Reeve, J., Anderson, L., Hill, C., . . Denehy, L. (2018). Preoperative physiotherapy for the prevention of respiratory complications after upper abdominal surgery: Pragmatic, double blinded, multicentre randomised controlled trial. BMJ, 360, j5916. https://doi.org/10.1136/bmj.j5916

Boulind, C. E., Yeo, M., Burkill, C., Witt, A., James, E., Ewings, P., . . Francis, N. K. (2012). Factors predicting deviation from an enhanced recovery programme and delayed discharge after laparoscopic colorectal surgery. Colorectal Disease, 14(3), e103-e110. https://doi.org/10.1111/j.14631318.2011.02799.x

Browning, L., Denehy, L., \& Scholes, R. L. (2007). The quantity of early upright mobilisation performed following upper abdominal surgery is low: An observational study. Australian Journal of Physiotherapy, 53(1), 47-52. https://doi.org/10.1016/S0004-9514(07)70061-2

Fagevik Olsén, M., Hahn, I., Nordgren, S., Lönroth, H., \& Lundholm, K. (1997). Randomized controlled trial of prophylactic chest physiotherapy in major abdominal surgery. British Journal of Surgery, 84(11), 1535-1538. https://doi.org/10.1111/j.1365-2168.1997.02828.x

Fagevik Olsén, M., Josefson, K., \& Lönroth, H. (1999). Chest physiotherapy does not improve the outcome in laparoscopic fundoplication and verticalbanded gastroplasty. Surgical Endoscopy, 13(3), 260-263. https://doi. org/10.1007/s004649900958

Feldheiser, A., Aziz, O., Baldini, G., Cox, B. P. B. W., Fearon, K. C. H., Feldman, L. S., . . C Carli, F. (2016). Enhanced Recovery After Surgery (ERAS) for gastrointestinal surgery, part 2: Consensus statement for anaesthesia practice. Acta Anaesthesiologica Scandinavica, 60(3), 289334. https://doi.org/10.1111/aas.12651

Fincham, J. E. (2008). Response rates and responsiveness for surveys, standards, and the Journal. American Journal of Pharmaceutical Education, $72(2), 43$.

Gustafsson, U. O., Hausel, J., Thorell, A., Ljungqvist, O., Soop, M., \& Nygren, J. (2011). Adherence to the enhanced recovery after surgery protocol and outcomes after colorectal cancer surgery. Archives of Surgery, 146(5), 571577. https://doi.org/10.1001/archsurg.2010.309.

Hanekom, S. D., Brooks, D., Denehy, L., Fagevik-Olsén, M., Hardcastle, T. C., Manie, S., \& Louw, Q. (2012). Reaching consensus on the physiotherapeutic management of patients following upper abdominal surgery: a pragmatic approach to interpret equivocal evidence. BMC Medical Informatics and Decision Making, 12, 5. https://doi. org/10.1186/1472-6947-12-5

Hijazi, Y., Gondal, U., \& Aziz, O. (2017). A systematic review of prehabilitation programs in abdominal cancer surgery. International Journal of Surgery, 39, 156-162. https://doi.org/10.1016/j.ijsu.2017.01.111

Katsura, M., Kuriyama, A., Takeshima, T., Fukuhara, S., \& Furukawa, T. A. (2015). Preoperative inspiratory muscle training for postoperative pulmonary complications in adults undergoing cardiac and major abdominal surgery. Cochrane Database of Systematic Reviews, 10 , CD010356. https://doi.org/10.1002/14651858.CD010356.pub2 
Kendall, F., Oliveira, J., Peleteiro, B., Pinho, P., \& Bastos, P. T. (2018). Inspiratory muscle training is effective to reduce postoperative pulmonary complications and length of hospital stay: A systematic review and metaanalysis. Disability and Rehabilitation, 40(8), 864-882. https://doi.org/10.1 080/09638288.2016.1277396

Loughney, L., West, M. A., Kemp, G. J., Grocott, M. P., \& Jack, S. (2015). Exercise intervention in people with cancer undergoing adjuvant cancer treatment following surgery: A systematic review. European Journal of Surgical Oncology, 41(12), 1590-1602. https://doi.org/10.1016/j. ejso.2015.08.153

Mackay, M., Ellis, E., \& Johnston, C. (2005). Randomised clinical trial of physiotherapy after open abdominal surgery in high risk patients. Australian Journal of Physiotherapy, 51(3), 151-159. https://doi. org/10.1016/S0004-9514(05)70021-0

Makhabah, D. N., Martino, F., \& Ambrosino, N. (2013). Peri-operative physiotherapy. Multidisciplinary Respiratory Medicine, 8(1), 4. https://doi. org/10.1186/2049-6958-8-4

Mans, C. M., Reeve, J. C., \& Elkins, M. R. (2015). Postoperative outcomes following preoperative inspiratory muscle training in patients undergoing cardiothoracic or upper abdominal surgery: A systematic review and meta analysis. Clinical Rehabilitation, 29(5), 426-438. https://doi. org/10.1177/0269215514545350

Moran, J., Guinan, E., McCormick, P., Larkin, J., Mockler, D., Hussey, J., . . . Wilson, F. (2016). The ability of prehabilitation to influence postoperative outcome after intra-abdominal operation: A systematic review and meta-analysis. Surgery, 160(5), 1189-1201. https://doi.org/10.1016/j. surg.2016.05.014

O'Doherty, A., West, M., Jack, S., \& Grocott, M. (2013). Preoperative aerobic exercise training in elective intra-cavity surgery: a systematic review. British Journal of Anaesthesia, 110(5), 679-689. https://doi.org/10.1093/bja/ aes514

Owen, R. M., Perez, S. D., Lytle, N., Patel, A., Davis, S., Lin, E., \& Sweeney, J. F. (2013). Impact of operative duration on postoperative pulmonary complications in laparoscopic versus open colectomy. Surgical Endoscopy, 27(10), 3555-3563. https://doi.org/10.1007/s00464-013-2949-9.

Pashikanti, L., \& Von Ah, D. (2012). Impact of early mobilization protocol on the medical-surgical inpatient population: An integrated review of literature. Clinical Nurse Specialist, 26(2), 87-94. https://doi.org/10.1097/ NUR.0b013e31824590e6

Pasquina, P., Tramèr, M., Granier, J., \& Walzer, B. (2006). Respiratory physiotherapy to prevent pulmonary complications after abdominal surgery: a systematic review. Chest, 130(6), 1887-1899. https://doi org/10.1378/chest.130.6.1887

Pasquina, P., Tramèr, M., \& Walder, B. (2003). Prophylactic respiratory physiotherapy after cardiac surgery: Systematic review. BMJ, 327, 1379. https://doi.org/10.1136/bmj.327.7428.1379

Patman, S. (2018). Critically appraised paper: Preoperative physiotherapy education halved postoperative pulmonary complications in patients after upper abdominal surgery [commentary]. Journal of Physiotherapy, 64(3), 195. https://doi.org/10.1016/j.jphys.2018.04.007

Patman, S., Bartley, A., Ferraz, A., \& Bunting, C. (2017). Physiotherapy in upper abdominal surgery - what is current practice in Australia? Archives of Physiotherapy, 7, 11. https://doi.org/10.1186/s40945-017-0039-3

Reeve, J., \& Boden, I. (2016). The physiotherapy management of patients undergoing abdominal surgery. New Zealand Journal of Physiotherapy 44(1), 33-49. https://doi.org/10.15619/NZJP/44.1.05

Reeve, J., Nicol, K., Stiller, K., McPherson, K., Birch, P., Gordon, I., \& Denehy, L. (2010). Does physiotherapy reduce the incidence of postoperative pulmonary complications following pulmonary resection via open thoracotomy? A preliminary randomised single-blind clinical trial. European Journal of Cardio-Thoracic Surgery, 37 (5), 1158-1166. https:// doi.org/10.1016/j.ejcts.2009.12.011
Samnani, S. S., Umer, M. F., Mehdi, S. H., \& Farid, F. N. (2014). Impact of preoperative counselling on early postoperative mobilization and its role in smooth recovery. International Scholarly Research Notices, 2014, 250536 https://doi.org/10.1155/2014/250536

Scholes, R., Denehy, L., Sztendur, E., \& Browning, L. (2006). A survey of Australian physiotherapy management of patients having abdominal surgery. Australian Journal of Physiotherapy, 52(1), S26-\$27.

Shander, A., Fleisher, L. A., Barie, P. S., Bigatello, L. M., Sladen, R. N., \& Watson, C. B. (2011). Clinical and economic burden of postoperative pulmonary complications: Patient safety summit on definition, riskreducing interventions, and preventive strategies. Critical Care Medicine, 39(9), 2163-2172. https://doi.org/10.1097/CCM.0b013e31821f0522

Silva, Y. R., Li, S. K., \& Rickard, M. J. (2013). Does the addition of deep breathing exercises to physiotherapy-directed early mobilisation alter patient outcomes following high-risk open upper abdominal surgery? Cluster randomised controlled trial. Physiotherapy, 99(3), 187-193. https:// doi.org/10.1016/j.physio.2012.09.006

Smith, P. R., Baig, M. A., Brito, V., Bader, F., Bergman, M. I., \& Alfonso, A. (2010). Postoperative pulmonary complications after laparotomy. Respiration, 80(4), 269-274. https://doi.org/10.1159/000253881.

Snowdon, D., Haines, T. P., \& Skinner, E. H. (2014). Preoperative intervention reduces postoperative pulmonary complications but not length of stay in cardiac surgical patients: A systematic review. Journal of Physiotherapy, 60(2), 66-77. https://doi.org/10.1016/j.jphys.2014.04.002

Spanjersberg, W., van Sambeeck, J., Bremers, A., Rosman, C., \& van Laarhoven, C. (2015). Systematic review and meta-analysis for laparoscopic versus open colon surgery with or without an ERAS programme. Surgical Endoscopy, 29(12), 3443-3453. https://doi. org/10.1007/s00464-015-4148-3

Stethen, T. W., Ghazi, Y. A., Heidel, R. E., Daley, B. J., Barnes, L., Patterson, D., \& McLoughlin, J. M. (2018). Walking to recovery: The effects of missed ambulation events on postsurgical recovery after bowel resection. Journal of Gastrointestinal Oncology, 9(5), 953-961. https://doi.org/10.21037/ jgo.2017.11.05

Tew, G. A., Ayyash, R., Durrand, J., \& Danjoux, G. R. (2018). Clinical guideline and recommendations on pre-operative exercise training in patients awaiting major non-cardiac surgery. Anaesthesia, 73(6), 750-768. https:// doi.org/doi:10.1111/anae.14177

Veldcamp, R., Kuhry, E., Hop, W. C., Jeekel, J., Kazemier, G., Bonjer, H. J., COlon cancer Laparoscopic or Open Resection Study Group. (2005). Laparoscopic surgery versus open surgery for colon cancer: Short-term outcomes of a randomised trial. The Lancet Oncology, 6(7), 477-484 https://doi.org/10.1016/\$1470-2045(05)70221-7

West, M. A., Loughney, L., Lythgoe, D., Barben, C. P., Sripadam, R., Kemp, G. J., . . Jack, S. (2015). Effect of prehabilitation on objectively measured physical fitness after neoadjuvant treatment in preoperative rectal cancer patients: a blinded interventional pilot study. British Journal of Anaesthesia, 114(2), 244-251. https://doi.org/10.1093/bja/aeu318

West, M. A., Lythgoe, D., Barben, C. P., Noble, L., Kemp, G. J., Jack, S., \& Grocott, M. P. (2014). Cardiopulmonary exercise variables are associated with postoperative morbidity after major colonic surgery: A prospective blinded observational study. British Journal of Anaesthesia, 112(4), 665671. https://doi.org/10.1093/bja/aet408

West, M. A., Parry, M. G., Lythgoe, D., Barben, C. P., Kemp, G. J., Grocott, M P. \& Jack, S. (2014). Cardiopulmonary exercise testing for the prediction of morbidity risk after rectal cancer surgery. British Journal of Surgery, 101(9), 1166-1172. https://doi.org/10.1002/bjs.9551

West, M. A., Wischmeyer, P. E., \& Grocott, M. P. W. (2017). Prehabilitation and nutritional support to improve perioperative outcomes. Current Anesthesiology Reports, 7(4), 340-349. https://doi.org/10.1007/s40140017-0245-2 